\title{
Serial or parallel processing in dual tasks: What is more effortful?
}

\author{
CAROLA LEHLE, MARCO STEINHAUSER, AND RONALD HÜBNER \\ Universität Konstanz, Konstanz, Germany
}

\begin{abstract}
Recent studies indicate that dual tasks can be performed with a serial or parallel strategy and that the parallel strategy is preferred even if this implies performance costs. The present study investigates the hypothesis that parallel processing is favored because it requires less mental effort compared to serial processing. A serial or parallel processing strategy was induced in a sample of 28 healthy participants. As measures of mental effort, we used a rating as well as heart rate (HR) and electrodermal activity. Parallel processing again showed performance costs relative to serial, whereas serial processing was judged as more effortful. Also tonic HR and phasic HR deceleration were increased with a serial strategy. Thus the preference for a parallel strategy in dual tasks likely reflects a compromise between optimizing performance and minimizing the amount of mental effort. This aspect is neglected in current dual task accounts so far.
\end{abstract}

Descriptors: Normal volunteers, Cognition and motivation, Electrodermal, Heart rate

Performing dual tasks, that is, performing two tasks at the same time or in close succession, typically leads to costs compared to a single task situation (Carrier \& Pashler, 1995; Logan \& Gordon, 2001; Pashler, 1994a). This indicates that the tasks have to compete for a limited capacity in the human information processing system. While much effort has been spent to prove that this always leads to a strict serial processing (Pashler, 1994b; Pashler \& Johnston, 1989), recently, an increasing number of studies has provided evidence that the processing capacity can be shared between the tasks in a graded fashion (e.g., Hübner \& Lehle, 2007; Miller, Ulrich, \& Rolke, in press; Tombu \& Jolicoeur, 2005). This means that participants can allocate a certain amount of capacity to one task while performing - with the remaining capacity - another task in parallel. In other words, dual tasks can be processed with either a more serial or a more parallel processing strategy. ${ }^{1}$ If people are free to choose between different degrees of parallel processing, an important question is which strategy they prefer and for what reason.

According to most dual-task theories, a serial strategy should be preferred because it minimizes confusion and crosstalk between the tasks (e.g., Logan \& Gordon, 2001). Crosstalk in dual tasks arises, for instance, on the level of response categories when both tasks make use of the same responses. In this case, a congruency effect is observable, i.e., if one task is associated with a

We thank Karen Grewen, Werner Sommer, and two anonymous reviewers for their valuable comments on an earlier version of this paper.

Address reprint requests to: Carola Lehle, Universität Konstanz, Fachbereich Psychologie, Fach D29, D-78457 Konstanz, Germany. Email: carola.lehle@uni-konstanz.de

'To decide whether parallel processing in dual tasks is possible during all stages or whether some processing units constitute a structural bottleneck is beyond the scope of the present study. different response than the other, performance is usually worse than if both tasks are mapped to the same response. However, up to now, it is unclear whether a serial strategy is really more optimal under these conditions. Some researchers assume that participants still prefer a parallel processing strategy - at least at short stimulus onset asynchronies (Miller et al:, in press).

In previous studies (Hübner \& Lehle, 2007; Lehle \& Hübner, in press), we investigated processing strategies in dual tasks with the psychological refractory period paradigm (PRP; Welford, 1952), in which the stimulus of the second task appears before the processing of the first task is completed. As a result, we found that the participants showed a strong tendency to process those tasks in parallel. At first sight, one might suppose that such a strategy was chosen because parallel processing leads to a benefit in performance. However, the opposite was the case. Increased parallel processing was accompanied by longer response times and higher error rates. We thus came to the conclusion that the participants were either mistaken to believe that parallel processing is beneficial in dual tasks or that they preferred a parallel strategy for a different reason. But what reason could that be?

Presumably, it is not overt performance that the participants intend to optimize by processing dual tasks in parallel. According to Hockey (1997), analyses of task performance need to take into account not only overt performance, such as response times and error rates, but also trade-offs among the participant's goals and strategies, and the amount of mental effort that is needed to achieve the goals (e.g., Steinhauser, Maier, \& Hübner, 2007). These considerations are based on Kahneman's thesis that effectiveness and efficiency of performance should be differentiated: "Effectiveness is a measure of the quality of performance, while efficiency is the relation between the quality of performance and the effort invested in it" (Kahneman, 1973, p. 181). 
Although many recent studies (e.g., Ruthruff, Pashler, \& Hazeltine, 2003; Ruthruff, Pashler, \& Klaassen, 2001; Tombu \& Jolicoeur, 2004, 2005) demonstrated that processing two tasks concurrently means that both tasks have to access limited resources (i.e., the "central capacity" or the "central bottleneck"), and, furthermore, that parallel processing might lead to increased costs in overt performance, none of these studies also examined mental effort. Accordingly, it is entirely unknown so far which role mental effort plays in the motivation to prefer either a serial or a more parallel processing strategy in dual tasks.

A strategy of serial processing might be more effortful in dual tasks with the PRP paradigm because it requires inhibiting the processing of the second stimulus for a relatively short time during the first task and then resuming it for the second task. That processing of previously inhibited stimuli is costly has been shown in studies of negative priming (e.g., Tipper, 1985; Tipper \& Cranston, 1985) and inhibition of return (e.g., Posner \& Cohen, 1984; Tipper, Weaver, Jerreat, \& Burak, 1994). By coprocessing the stimuli from both tasks right from the beginning of a trial, participants can avoid the effortful procedure of first inhibiting and then resuming the processing of the other stimulus. In this case the strategy of parallel processing would be a compromise between optimizing the performance and minimizing the processing effort-thus increasing the efficiency.

Whether parallel processing is indeed more efficient in dual tasks than serial processing was investigated in the present study. Because previous results have shown that participants are able to vary the degree of parallel processing in a graded fashion according to specific instructions (Lehle \& Hübner, in press; for a review see Navon \& Gopher, 1979), we also used this method in the present study. That is, participants were instructed to process dual tasks either in a serial or in a more parallel mode. Overt performance was analyzed by measuring response times and error rates. However, additional measures were needed to quantify the processing effort in the serial compared to the parallel instruction condition.

To obtain this objective, we asked the participants to rate the amount of mental effort they experienced during conducting the tasks in the serial compared to the parallel mode. If a parallel processing strategy is consciously applied as a trade-off between mental effort on the one side and overt performance on the other, participants should be able to estimate the degree of effort required in the different conditions (Naccache, Dehaene, Cohen, Habert, Guichart-Gomez, Galanaud, \& Willer, 2005). If this is not the case, then this would suggest that the compromise between effort and performance is either not consciously accessible or that other reasons might be responsible for the tendency to process the tasks in parallel.

In addition, we also used psychophysiological correlates of mental effort. It has frequently been shown that processes paralleling the experience of mental effort involve increased autonomic activation (see Beatty, 1982; Cacioppo, Tassinary, \& Berntson, 2000; Sourkes, 2006 for reviews). Specifically, it has been demonstrated that mental effort is associated with increased heart rate (e.g., Boutcher \& Boutcher, 2006; Brown, Szabo, \& Seraganian, 1988; Carroll \& Turner, 1986; Steptoe, Moses, Mathews, \& Edwards, 1990) and with increased electrodermal activity (e.g., Collet, Petit, Priez, \& Dittmar, 2005; Kahneman, 1973; Lang, Bradley, \& Cuthbert, 1990; Naccache et al., 2005). Therefore, we recorded the electrocardiogram and the electrodermal activity of the participants while they performed the dual tasks.

We predicted that, if serial processing is more effortful compared to parallel processing in dual tasks, heart rate and skin conductance should be higher under the serial than under the parallel instruction condition.

\section{Method}

\section{Participants}

Twenty-eight participants ( 21 female, 7 male) between 19 and 38 years of age ( $M=24$ years) with normal or corrected-to-normal vision participated in the study. Participants were recruited at the Universität Konstanz and were paid $5 €$ per hour. Whereas electrodermal activity was measured in all participants, heart rate was, due to a temporary failure of the recording equipment, recorded only in a sub-sample of 18 participants.

\section{Apparatus}

The stimuli were presented on a $21^{\prime \prime}$-monitor connected to a personal computer (PC) with a resolution of $1280 \times 768$ pixels, and a refresh rate of $85 \mathrm{~Hz}$. The software controlling stimulus presentation and registration of the behavioral responses was programmed in $\mathrm{C}++$ (Microsoft Inc.). Responses had to be given by pressing either the "shift left" or the "shift right" button on a standard PC keyboard.

\section{Stimuli}

The stimulus set consisted of eight numerals $(1,2,3,4,6,7,8$, and 9). The height of the stimuli subtended a visual angle of $2^{\circ}$ at a viewing distance of $72 \mathrm{~cm}$, and their width was about $1.36^{\circ}$, depending on the specific digit. The stimulus $\left(S_{1}\right)$ for the first task was presented at the center of the screen, whereas the stimulus $\left(\mathrm{S}_{2}\right)$ for the second task consisted of two copies of a numeral, which were presented left and right of $S_{1}$ at an eccentricity of $1.18^{\circ}$. Altogether three digits appeared on each trial with $S_{1}$ at the center position. $S_{1}$ and $S_{2}$ were always different.

\section{Procedure}

The task for the participants was to judge the parity (odd, even) of the stimuli. For the first and the second task, one of two buttons had to be pressed with the right hand. For an "even" number, participants had to push the left button with the index finger, for an "odd" number the right button with the middle finger, respectively. Each trial started with the appearance of a fixation cross for $400 \mathrm{~ms}$. After the presentation of a blank screen of 600 ms duration, both $S_{1}$ and $S_{2}$ appeared in white color on a black background.

$S_{1}$ and $S_{2}$ were congruent on half of the trials, i.e., had the same parity, and were incongruent on the other half, i.e., had the opposite parity. Participants always had to respond to $S_{1}$ first and subsequently to $S_{2}$. The stimuli remained on the screen until the participants' responses had occurred. Six seconds after the last response, the fixation cross for the next trial appeared. This long intertrial-interval was necessary to reliably assign skin conductance changes to individual stimuli. Trials with a wrong answer in either one or both responses were categorized as errors.

Participants received specific instructions on how they had to allocate their capacity within a block. There were two block types: For one type, the participants were instructed to allocate their capacity only to $S_{1}$ first and to ignore $S_{2} . S_{2}$ processing should not start before the first response had been selected. For the other block type, the participants were instructed to distribute their capacity also to $S_{2}$ from the beginning of a trial. 
Four successive blocks of one instruction type alternated with four blocks of the other type, whereas the instruction order was balanced across participants. Altogether there were 10 blocks with 32 trials each. Accordingly, participants performed 80 trials under each of the four experimental conditions (serial/congruent, serial/incongruent, parallel/congruent, parallel/incongruent). Before the specific instructions were given, there was a training block to familiarize the participants with the basic task. The whole experimental session took about 90 minutes.

\section{Rating of General Procedure and Effort}

Participants had to fill in a questionnaire subsequent to the experiment, which we had constructed for the present purpose. One part of the questionnaire consisted of general questions about the procedure such as enjoyment, tiredness, and possible artifacts during the experiment (e.g., changing the seating position or speaking loudly during the measures). In the other part, participants had to rate the level of effort they experienced during the experiment retrospectively and separately for the serial and the parallel condition. This part was titled by the question "How effortful do you judge the different conditions?" The answers were formulated as follows: "I found the serial condition ... "-respectively-"I found the parallel condition ... "and choices could be made on a Likert scale ranging from $1=$ "very little effortful" to $6=$ "extremely effortful."

\section{Psychophysiological Recording}

The electrocardiogram was recorded at a sampling rate of $200 \mathrm{~Hz}$ using two $\mathrm{Ag} / \mathrm{AgCL}$ electrodes placed on the left and right ventral forearm. The electrodermal activity was recorded at a sampling rate of $200 \mathrm{~Hz}$ using $30 \mathrm{~mm}^{2}$ unpolarizable $\mathrm{Ag} / \mathrm{AgCl}$ electrodes placed on the thenar and hypothenar surfaces of the participant's left hand. The amplifier (Biopac, GSR100C, Biopac Systems, Inc., Goleta, CA) used a constant voltage of $0.5 \mathrm{~V}$ DC. Prior to having the electrodes attached, participants were requested to wash their hands; subsequently, electrode sites for the measurement of the electrocardiogram and the electrodermal activity were prepared by cleaning the skin with ethyl alcohol $(70 \%)$. The electrodes were filled with an isotonic conductive gel (Biopac, Gel 101) to improve sensor-skin contact. The recording took place in a quiet and dimly lit chamber. Participants were requested to sit quietly during the experimental blocks.
Psychophysiological Data Analysis

Unless noted otherwise, data pre-processing and analyses were computed using MatLab (The Mathworks, Inc., Natick, MA).

Electrocardiogram. $R$-peaks were detected off-line with an accuracy of $5 \mathrm{~ms}$ using AcqKnowledge 3.7 .3 (Biopac). A continuous heart rate (HR) was obtained by transforming inter-beat intervals into a continuous signal (cf. Koers, Mulder, \& van der Veen, 1999). This served as the base for calculating tonic and phasic HR measures. Tonic HR was defined as the average HR in a $2000 \mathrm{~ms}$ time window directly preceding the stimulus onset. In this way, tonic HR should be independent from phasic HR changes induced by the stimulus. To analyze phasic HR changes, we averaged across segments from $0 \mathrm{~ms}$ to $3000 \mathrm{~ms}$ following stimulus onset. These segments were corrected by a baseline which corresponded to the mean HR in the $1000 \mathrm{~ms}$ pre-stimulus time window. For the statistical analysis, we determined the amplitude and the latency of the minimum $H R$ within this time window for each trial. Trials with response errors and artifacts were excluded from further analyses. A segment was regarded as being contaminated by an artifact if the standard deviation of HR exceeded a criterion that was determined separately for each participant by means of visually inspecting the distribution of standard deviations across trials. The proportions of trials contaminated by artifacts under the four conditions were $3.12 \%$ (congruent/serial), 3.03\% (incongruent/serial), $2.44 \%$ (congruent/parallel), and $2.40 \%$ (incongruent/parallel). The resulting trial numbers are shown in Table 1.

Electrodermal Activity: As a measure of electrodermal activity, we computed event-related skin conductance responses (SCR). A signal change of $0.2 \mu$ Siemens occurring within $3 \mathrm{~s}$ after the stimulus was classified as an event-related SCR. For each condition, the relative frequency of trials containing an SCR as well as the mean amplitude of these SCRs was derived. Again, trials with errors or artifacts were excluded from further analyses. The data of one participant had to be excluded due to an extremely high number of artifacts. Apart from that, only few segments were excluded based on visual inspection (less than $1 \%$ of trials). The resulting trial numbers are provided in Table 1.

Table 1. Mean Response Times (RTs) in ms and Error Rates in \% of First and Second Responses as well as Mean Trial Numbers for the Two Subsamples Used in the Analysis of Electrodermal Activity (EDA) and Heart Rate (HR) Data

\begin{tabular}{|c|c|c|c|c|c|}
\hline & \multicolumn{2}{|c|}{ Response 1} & \multicolumn{2}{|c|}{ Response 2} & \multirow{2}{*}{$\begin{array}{l}\text { Mean number of trials } \\
\text { in HR/EDA analysis }\end{array}$} \\
\hline & $\overline{\mathrm{RT}}$ & $\%$ error & RT & $\%$ error & \\
\hline \multicolumn{6}{|l|}{ HR sample $(n=18)$} \\
\hline Serial/congruent & 711 & 1.7 & 1085 & 3.3 & 61.6 \\
\hline Serial/incongruent & 785 & 2.8 & 1140 & 2.2 & 68.1 \\
\hline Parallel/congruent & 777 & 1.0 & 1017 & 3.2 & 68.6 \\
\hline Parallel/incongruent & 954 & 4.0 & 1178 & 4.6 & 64.7 \\
\hline \multicolumn{6}{|l|}{ EDA sample $(n=27)$} \\
\hline Serial/congruent & 727 & 1.8 & 1083 & 4.0 & 67.4 \\
\hline Serial/incongruent & 788 & 3.3 & 1118 & 2.9 & 73.6 \\
\hline Parallel/congruent & 774 & 1.2 & 1021 & 3.0 & 72.0 \\
\hline Parallel/incongruent & 925 & 6.3 & 1155 & 6.8 & 67.6 \\
\hline
\end{tabular}

Note: $\mathrm{RT}=$ response time (in $\mathrm{ms}$ ), $\mathrm{HR}=$ heart rate, $\mathrm{EDA}=$ electrodermal activity. 

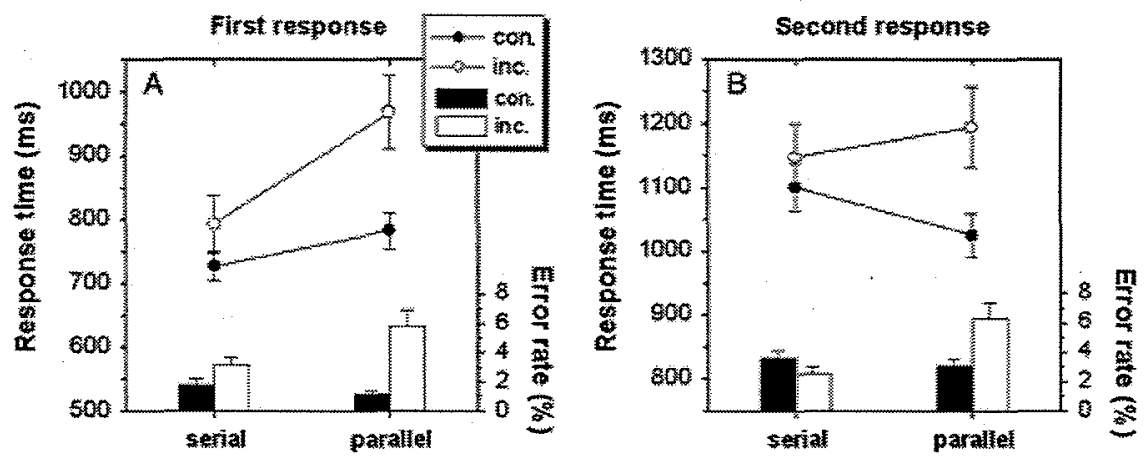

Figure 1. The behavioral data for the first (A) and for the second response (B). "Con." and "inc." denote the congruent and incongruent condition, respectively.

\section{Results}

\section{Behavioral Data}

Response times as well as error rates for the first and the second response were entered into a two-way ANOVA with repeated measurement on the variables Instruction (serial, parallel) and Congruency (congruent, incongruent). Response times were analyzed only for those trials on which both responses were correct. In the following, we report the results for the whole sample. However, the results were virtually identical when we considered only participants used for analyzing HR data and EDA data (see Table 1).

Response times of the first response showed significant main effects of Instruction, $F(1,27)=9.10, \eta^{2}=.25, p<.01$, and Congruency, $\quad F(1,27)=26.6, \quad \eta^{2}=.50, \quad p<.001$. Instruction and Congruency also interacted significantly, $F(1,27)=6.72$, $\eta^{2}=.20, p<.05$. Figure $1 \mathrm{~A}$ reveals that, with a parallel instruction, responses were generally slower and the effect of Congruency was strongly increased. A similar pattern emerged for the error rates of the first response. The main effect of instruction, i.e., a trend of more errors with the parallel instruction, just failed from being significant $F(1,27)=3.33, \eta^{2}=.11, p=.079$. Furthermore, there was a significant main effect of Congruency, $F(1,27)=23.4, \eta^{2}=.47, p<.001$, and an interaction between Instruction and Congruency, $F(1,27)=9.79, \eta^{2}=.27, p<.01$. Again, more errors and an increased effect of Congruency were evident with a parallel instruction.

The analysis of the response times of the second response revealed only a significant main effect of Congruency, $F(1$, $27)=16.0, \eta^{2}=.37, p<.001$, and an interaction of Congruency and Instruction, $F(1,27)=6.48, \eta^{2}=.19, p<.05$. Figure $1 \mathrm{~B}$ shows that this results from an increased effect of Congruency with a parallel instruction. The error rates for the second response showed a significant main effect of Instruction, $F(1,27)=5.36, \eta^{2}=.17, p<.05$, and a significant interaction between Instruction and Congruency, $F(1,27)=13.0, \eta^{2}=.33$, $p<.01$. Again, this mainly reflects the increased effect of Congruency with a parallel instruction.

\section{Effort-Rating Data}

The data from the effort rating in the questionnaire were analyzed by computing the mean values for the serial and the parallel condition. On average, participants experienced more effort under the serial $(M=3.9)$ than under the parallel instruction condition $(M=2.1)$. A test revealed this difference as highly significant, $t(27)=5.27, \eta^{2}=.51, p<.001$.

\section{Electrocardiogram}

Figure 2 shows the mean HR in a time window of $3000 \mathrm{~ms}$ before and after stimulus onset. As can be seen, there is a rather constant difference in HR between the serial and the parallel condition.

Because there seems to be no phasic HR change in anticipation of the stimulus that differs between parallel and serial conditions, we computed the tonic HR during an interval of $2000 \mathrm{~ms}$ preceding stimulus onset. The values of tonic $H R$ were then entered into a one-way ANOVA with repeated measurement on the variable Instruction (parallel, serial). Because tonic HR was defined in a pre-stimulus interval and thus could not be affected by the type of stimulus, Congruency was not considered in this analysis. As illustrated in Figure 3A, the tonic HR was higher in blocks with a serial instruction than in blocks with a parallel instruction, $F(1,17)=4.55, \eta^{2}=.21, p<.05$.

The averaged phasic HR change is illustrated in Figure 4, showing average wave forms for each combination of the variables Instruction (serial, parallel) and Congruency (congruent, incongruent). As evident, stimulus presentation is followed by a typical HR deceleration. To analyze this deceleration statistically, mean amplitudes and latency of minimum HR were

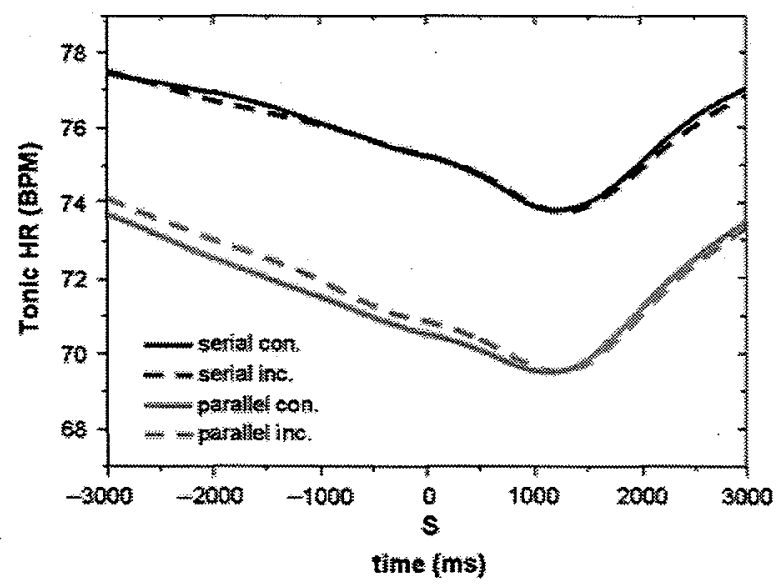

Figure 2. The time course of the tonic heart rate (HR) from $3000 \mathrm{~ms}$ before stimulus onset (S) until $3000 \mathrm{~ms}$ after stimulus onset. "Con." and "inc." denote the congruent and incongruent condition, respectively. 

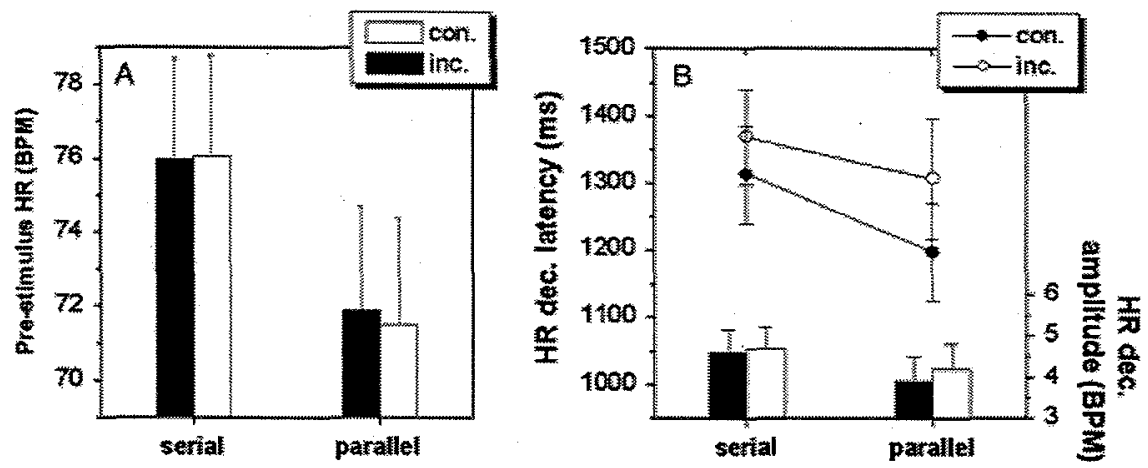

Figure 3. Tonic changes of the heart rate (HR) in the pre-stimulus interval of $2000 \mathrm{~ms}$ (A); latency and amplitude of the deceleration after stimulus onset (B). "Con." and "inc." denote the congruent and incongruent condition, respectively; "dec." refers to the deceleration of the HR.

entered into two-way ANOVAs with repeated measurement on the variables Instruction and Congruency. For the amplitudes, we obtained a significant main effect of Instruction, $F(1$, $17)=5.80, \eta^{2}=.25, p<.05$. As shown in Figure 3B, the amplitude of the HR deceleration was stronger in blocks with a serial instruction. Moreover, there was a tendency in blocks with a parallel instruction that the deceleration amplitude was higher on incongruent compared to congruent trials. ${ }^{2}$ For the latency of HR minima, we obtained a significant main effect of Congruency, $F(1,17)=7.66, \eta^{2}=.31, p<.05$, and a marginally significant interaction between Congruency and Instruction, $F(1$, $17)=3.30, \eta^{2}=.16, p=.087$. The minimum of the HR deceleration is influenced by Congruency more strongly in the parallel condition (see Figure 3B).

\section{Electrodermal Activity}

The analysis for SCR frequency revealed only a significant main effect of Congruency, $F(1,26)=4.29, \eta^{2}=.14, p<.05$. Figure 5 shows that this frequency was increased on incongruent trials. Although a congruency effect was present only in the parallel condition, the interaction between Instruction and Congruency did not reach significance $\left[F(1,26)=2.45, \eta^{2}=.09, p=.129\right]$. No significant effect on the SCR amplitude was detected $(F s<1)$. The mean amplitudes in the four conditions were $0.60 \mu$ Siemens (congruent/serial), $0.61 \mu$ Siemens (incongruent/serial), 0.63 $\mu$ Siemens (congruent/parallel), and $0.62 \mu$ Siemens (incongruent/ parallel).

\section{DISCUSSION}

The present study was conducted to investigate whether serial processing is more effortful compared to parallel processing in PRP-like dual tasks in which $S_{2}$ is presented before processing of $S_{1}$ is completed. Different degrees of parallel processing were realized by instructing the participants to perform dual tasks with either a serial or with a parallel processing strategy. Because response times and error rates cannot indicate the amount of effort invested in a task (cf. Hockey, 1997), our participants rated the degree of effort they experienced in the two conditions. If

${ }^{2}$ Please note that the differences in HR minimum in our conditions are only weakly reflected in Figure 4 . Since the minimum is at a different point in time on each trial, averaging blurs the differences in the minimum amplitudes. participants intentionally chose a parallel processing strategy in dual tasks because it is accompanied by less mental effort, they should be able to report on it. Moreover, psychophysiological measures were included in the present study to further compare the serial and the parallel instruction condition with respect to correlates of mental effort. Here, HR and event-related SCR were used because these measures are known to be increased with an increased mental effort (Boutcher \& Boutcher, 2006; Naccache et al., 2005).

First of all, the behavioral data replicated our previous results (Hübner \& Lehle, 2007; Lehle \& Hübner, in press). Response times and error rates were higher overall if they were performed with a parallel compared to a serial processing strategy. This confirms that parallel processing does not lead to a benefit in performance, but to increased costs. In the present study, of course, the participants were instructed to adopt either a serial or a parallel processing strategy. However, in our previous studies including dual task conditions without specific instructions (Hübner \& Lehle, 2007; Lehle \& Hübner, in press), participants clearly preferred a parallel processing strategy and by that accepted the costs in performance. In view of these results, we

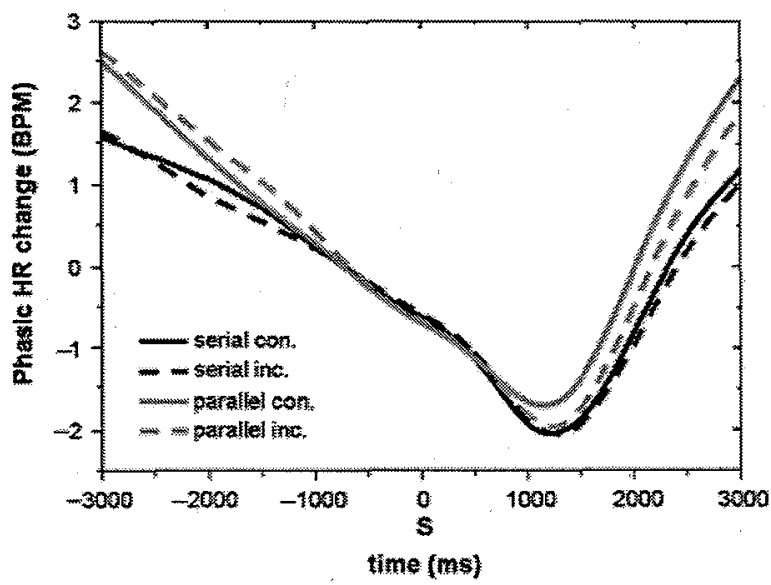

Figure 4. The time course of the phasic heart rate (HR) change from 3000 ms before stimulus onset (S) until $3000 \mathrm{~ms}$ after stimulus onset. "Con." and "inc." denote the congruent and incongruent condition, respectively. 


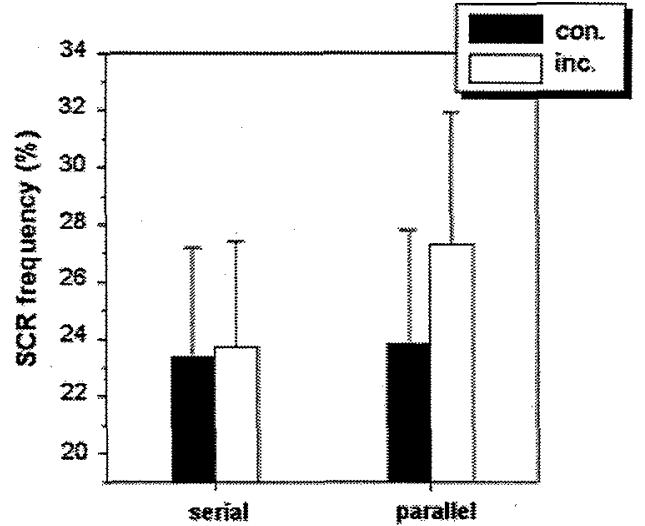

Figure 5. The mean frequencies of skin conductance responses (SCR) in the different conditions. "Con." and "inc." denote the congruent and incongruent condition, respectively.

hypothesized that the motivation to process dual tasks in parallel emerges from the intention to minimize mental effort.

The main question of the present study therefore was whether the performance costs with a parallel strategy are compensated by a reduced effort. Considering the effort rating data, this was indeed the case. In accordance with our hypothesis, participants rated the serial processing strategy as more effortful compared to the parallel processing strategy. Furthermore, the psychophysiological data seem to parallel this result. The tonic HR was considerably increased during the condition with a serial compared to a parallel processing strategy. Several studies so far have shown that in task conditions requiring more mental effort, tonic HR is increased compared to control conditions (e.g. Boutcher \& Boutcher, 2006; Brown et al., 1988; Hamer, Boutcher, \& Boutcher, 2003; Sammer, 1998; Steptoe et al., 1990). Apart from that, there was a phasic HR deceleration after stimulus onset in each condition. The amplitude as well as the latency of the HR deceleration was higher in blocks with a serial compared to blocks with a parallel instruction. Transient cardiac deceleration typically occurs with stimulus intake and reffects the engagement of attention to external stimuli (e.g., Jennings \& van der Molen 2002; Jennings, van der Molen, Brock, \& Somsen, 1991).

The question emerges whether our tonic HR measure is affected by phasic HR changes. Indeed, phasic effects evident with stimulus presentation might already start in the pre-stimulus interval, thus reflecting task preparation (cf. De Jong, 1995). For the present results, however, inspection of the time course in the pre-stimulus interval revealed a rather constant difference between the serial and the parallel instruction condition. This indicates that our tonic HR measure - which was defined as mean HR in a pre-stimulus interval - was not substantially influenced by phasic HR effects emerging during task preparation. Moreover, the stimulus-locked phasic effects observed after stimulus presentation even seem to counteract the differences obtained for the tonic HR: There was a stronger decrease in the phasic HR in the serial compared to the parallel condition, whereas the tonic HR was increased under the serial compared to the parallel instruction. Accordingly, phasic effects cannot account for the increased tonic HR in the serial condition.

The electrodermal activity appeared to be less infuenced by the processing strategy, i.e., there was no significant effect of the instruction condition on the frequency or on the amplitude of the SCRs. There was only a significant main effect of congruency on SCR frequency, i.e., more SCRs occurred on incongruent than on congruent trials. However, a clear trend in the data also indicated that most SCRs occurred on incongruent trials with a parallel processing strategy. Accordingly, the pattern of SCRs seems to reflect the amount of response conflict induced by the stimulus. This outcome corroborates previous results showing that the electrodermal reactivity is particularly sensitive to conflict on stimulus level (Naccache et al., 2005).

It has been frequently observed in the literature that $\mathrm{HR}$ and electrodermal reactivity are dissociable. This finding is usually explained by differences in autonomic response patterns (see Lacey, 1967) or by the hypothesis that cardiovascular and electrodermal adjustments are linked to different behavioral systems (Amodio, Master, Yee, \& Taylor, 2007; Fowles, 1988; Gray, 1987). Furthermore, the HR seems to be particularly sensitive for effort mobilization, whereas the electrodermal system is more influenced by conflict or aversive feedback (e.g., Tranel, 1983). The data of the present study further support this interpretation.

Taken together, the results of the present study are compatible with the hypothesis that serial dual-task processing is more effortful compared to parallel processing. But how can the increased mental effort be explained? Shielding one task from the other to minimize crosstalk and to achieve a serial processing strategy requires a strict focusing of attention. This means that, during the first task, the processing of the second stimulus has to be inhibited for a relatively short time interval and then to be resumed for the processing of the second task. It can be assumed that this requires a high amount of cognitive control (e.g., Posner \& Cohen, 1984; Tipper et al., 1994), presumably accompanied by increased mental effort.

One could object that, in the present study, only dual tasks were used where the task set was identical for the first and the second task. In this case, the crosstalk between the tasks and thus also the effort in shielding one task from the other could be particularly high. In a previous study including also dual tasks with different task sets, overt performance was less influenced by the other task, but the overall pattern of results was very similar (Lehle \& Hübner, in press). Participants also showed a tendency to strategic parallel processing, although it was more costly compared to serial processing.

The exact physiological mechanisms in the brain that lead to the characteristic autonomic reactions associated with mental effort are fairly unknown so far. However, mesio-frontal structures, including the anterior cingulate cortex, likely play a role in the generation of somatic signals in response to mental effort (e.g., Critchley, Mathias, Josephs, O'Doherty, Zanini, \& Dewar, 2003; Tranel \& Damasio, 1994). Moreover, the feeling of effort that the participants experienced could also be related to signals generated by the anterior cingulate cortex. It has been reported that a patient with a vast lesion in these structures had lost almost entirely the ability to experience and report a feeling of mental effort, although cognitive abilities and overt performance were preserved (Naccache et al., 2005).

To conclude, the present results indicate that not only overt performance but also the amount of mental effort should be taken into account to judge the efficiency of processing strategies. This aspect is largely neglected in current dual task accounts. Considering only overt performance, many theories come to the conclusion that serial processing is advantageous in dual tasks (e.g., Logan \& Gordon, 2001; Navon \& Miller, 1987; 
Navon \& Miller, 2002). In contrast, the present data indicate that different processing strategies in dual tasks also produce different levels of experienced effort. This is likely to play an important role in the choice of one strategy (i.e., parallel processing) over the other.

When participants spontaneously adopt a parallel processing strategy (Hübner \& Lehle, 2007; Lehle \& Hübner, in press), they seem to optimize their behavior not only with respect to overt performance, but also with respect to mental effort. Only if this aspect is also taken into account, an adequate consideration of serial compared to parallel processing strategies in dual tasks seems possible. Future research should further investigate the role of mental effort in strategic considerations during cognitive tasks. Also, additional psychophysiological data (e.g., event-related potentials) would be desirable to investigate these effects in more detail.

\section{REFERENCES}

Amodio, D. M., Master, S. L Yee, C. M. \& Taylor, S. E (2007) Neurocognitive components of the behavioral inhibition and activation systems: Implications for theories of self-regulation. Psychophysiology, 45, 11-19.

Beatty, J. (1982). Task-evoked pupillary responses, processing load, and the structure of processing resources. Psychological Bulletin, 91, 276-292.

Boutcher, Y. N., \& Boutcher, S. H. (2006). Cardiovascular response to Stroop: Effect of verbal response and task difficulty. Biological Psychology, 73, 235-241.

Brown, T. G., Szabo, A., \& Seraganian, P. (1988). Physical versus psychological determinants of heart rate reactivity to mental arithmetic. Psychophysiology, 25, 532-537.

Cacioppo, J., Tassinary, L., \& Berntson, G. (2000). Handbook of psychophysiology. Cambridge: University Press.

Carrier, L. M., \& Pashler, H. (1995). Attentional limits in memory retrieval. Journal of Experimental Psychology: Learning, Memory, and Cognition, 21, 1339-1348.

Carroll, D., \& Turner, J. R. (1986). Heart rate and oxygen consumption during active psychological challenge: The effects of level of difficulty Psychophysiology, 23, 174-181

Collet, C., Petit, C., Priez, A., \& Dittmar, A. (2005). Stroop color-word test, arousal, electrodermal activity and performance in a critical driving situation. Biological Psychology, 69, 195-203.

Critchley, H. D., Mathias, C. J., Josephs, O., O'Doherty, J., Zanini, S., \& Dewar, B. K. (2003). Human cingulate cortex and autonomic control: Converging neuroimaging and clinical evidence. Brain, 126, 21392152

De Jong, R. (1995). The role of preparation in overlapping-task performance. Quarterly Journal of Experimental Psychology [A] $48,2-25$.

Fowles, D. (1988). Psychophysiological and psychopathology: A motivational approach. Psychophysiology, 25, 373-391.

Gray, J. A. (1987). The psychology of fear and stress. Cambridge, UK Cambridge University Press.

Hamer, M., Boutcher, Y. H., \& Boutcher, S. H. (2003). The role of cardiopulmonary baroreceptors during the forearm vasodilatation response to mental stress in humans. Psychophysiology, 40, 249-253

Hockey, G. R. J. (1997). Compensatory control in the regulation of human performance under stress and high workload: A cognitiveenergetical framework. Biological Psychology, 45, 73-93.

Hübner, R., \& Lehle, C. (2007). Strategies of tlanker coprocessing in single and dual tasks. Journal of Experimental Psychology: Human Perception and Performance, 33, 103-123.

Jennings, J. R., \& van der Molen, M. W. (2002). Cardiac timing and the central regulation of action. Psychological Research, 66, 337-349.

Jennings, J. R., van der Molen, M. W., Brock, K., \& Somsen, R. J. M. (1991). Response inhibition initiates cardiac deceleration: Evidence from a sensory-motor compatibility paradigm. Psychophysiology, 28 , $72-85$.

Kahneman, D. (1973). Attention and effort. Englewood Cliffs, NJ: Prentice Hall.

Koers, G., Mulder, L. J. M. \& van der Veen, F. M. (1999). The computation of evoked heart rate and blood pressure. Journal of Psychophysiology, 13, 83-91.

Lacey, J. I. (1967). Somatic response patterning and stress: Some revisions of activation theory. In M. H. Appley \& R. Trumbull (Eds.), Psychological stress: Issues in research (pp. 14-42). New York: Appleton-Century-Crofts.

Lang, P. J., Bradley, M. M., \& Cuthbert, B. N. (1990). Emotion, attention, and the startle reflex. Psychological Review, 97, 377-395.
Lehle, C., \& Hübner, R. (in press). Strategic capacity sharing between dual tasks: Evidence from tasks with the same and with different task sets. Psychological Research.

Logan, G. D., \& Gordon, R. D. (2001). Executive control of visual attention in dual-task situations. Psychological Review, 108, 393- 434.

Miller, J., Ulrich, R., \& Rolke, B. (in press). On the optimality of serial and parallel processing in the psychological refractory period paradigm: Effects of the distribution of stimulus onset asynchronies. Cognitive Psychology.

Naccache, L., Dehaene, S., Cohen, L., Habert, M.-O., Guichart-Gomez, E., Galanaud, D., \& Willer, J.-C. (2005). Effortless control: Executive attention and conscious feeling of mental effort are dissociable Neuropsychologia, 43, 1318-1328.

Navon, D., \& Gopher, D. (1979). On the economy of the humanprocessing system. Psychological Review, 86, 214-255.

Navon, D., \& Miller, J. (1987). Role of outcome conflict in dual-task interference. Journal of Experimental Psychology: Human Perception and Performance, 13, 435-448.

Navon, D., \& Miller, J. (2002). Queuing or sharing? A critical evaluation of the single-bottleneck notion. Cognitive Psychology, 44, 193-251

Pashler, H. (1994a). Dual-task interference in simple tasks: Data and theory. Psychological Bulletin, 116, 220-244.

Pashler, H. (1994b). Graded capacity-sharing in dual-task interference? Journal of Experimental Psychology: Human Perception and Performance, $20,330-342$.

Pashler, H., \& Johnston, J. C. (1989). Chronometric evidence for central postponement in temporally overlapping tasks. Quarterly Journal of Experimental Psychology: Human Experimental Psychology, 41, $19-45$.

Posner, M. I., \& Cohen, Y. (1984). Components of visual orienting. In H. Bouma \& D. G. Bouwhuis (Eds.), Attention and performance $X$. Control of language processes (Vol. 32, pp. 531-556). London: Lawrence Erlbaum Associates.

Ruthruff, E., Pashler, H. E., \& Hazeltine, E. (2003). Dual-task interference with equal task emphasis: Graded capacity sharing or central postponement? Perception \& Psychophysics, 65, 801-816.

Ruthruff, E., Pashler, H. E., \& Klaassen, A. (2001). Processing bottlenecks in dual-task performance: Structural limitation or strategic postponement? Psychonomic Bulletin \& Review, 8, 73-80.

Sammer, G. (1998). Heart period variability and respiratory changes associated with physical and mental load: Non-linear analysis. Ergonomics, $41,746-755$.

Sourkes, T. L. (2006). On the energy cost of mental effort. Journal of the History of Neurosciences, 15, 31-47.

Steinhauser, M., Maier, M., \& Hübner, R. (2007). Cognitive control under stress. Psychological Science, 18, 540-545.

Steptoe, A., Moses, J., Mathews, A., \& Edwards, S. (1990). Aerobic fitness, physical activity and psychophysiological reactions to mental tasks. Psychophysiology, 27, 264-274.

Tipper, S. P. (1985). The negative priming effect: Inhibitory priming by ignored objects. The Ouarterly Journal of Experimental Psychology A: Human Experimental Psychology, 37 A, 571-590.

Tipper, S. P., \& Cranston, M. (1985). Selective attention and priming: Inhibitory and facilitory effects of ignored primes. Quarterly Journal of Experimental Psychology A: Human Experimental Psychology, $37 A, 591-611$.

Tipper, S. P., Weaver, B., Jerreat, L. M., \& Burak, A. L. (1994). Objectbased and environment-based inhibition of return of visual attention. 
Journal of Experimental Psychology: Human Perception and Performance, 20, 478-499.

Tombu, M., \& Jolicoeur, P. (2004). Virtually no evidence for virtually perfect time-sharing. Journal of Experimental Psychology: Human Perception and Performance, 30, 795-810.

Tombu, M., \& Jolicoeur, P. (2005). Testing the predictions of the central capacity sharing model. Journal of Experimental Psychology: Human. Perception and Performance, 31, 790-802.

Tranel, D. (1983). The effects on monetary incentive and frustrative nonreward on heart rate and electrodermal activity. Psychophysiology, 20, 652-657.
Tranel, D., \& Damasio, H. (1994). Neuroanatomical correlates of electrodermal skin conductance responses. Psychophysiology, 31, 427438 .

Welford, A. T. (1952). The 'psychological refractory period' and the timing of high-speed performance: A review and a theory. British Journal of Psychology, 43, 2-19. 